\title{
NUEVOS DATOS PARA LAS ESPECIES DE CERATININI DE ESPAÑA Y PORTUGAL, CON UNA CLAVE PARA SU IDENTIFICACIÓN (HYMENOPTERA, APOIDEA, XYLOCOPINAE)
}

\author{
M. Terzo* y F. J. Ortiz-Sánchez**
}

\begin{abstract}
RESUMEN
Se ha realizado una revisión de las especies españolas y portuguesas de la tribu Ceratinini, basada en el estudio tanto de diversas colecciones entomológicas como de la información bibliográfica disponible. En total, 13 especies del género Ceratina Latreille, 1802 habitan en la Península Ibérica, más las Islas Baleares y Canarias. Las más frecuentes, según el conocimiento actual, son $C$. cucurbitina (Rossi, 1792) y $C$. cyanea (Kirby, 1802), mientras que la más rara es C. albosticta Cockerell, 1931 que, con un solo registro, es nueva para nuestra fauna, al igual que C. gravidula Gerstaecker, 1869. Se elimina del catálogo de especies ibéricas a C. acuta Friese, 1896 y C. loewi Gerstaecker, 1869, mencionadas por primera vez por Dusmet (1923), pero que seguramente fueron mal identificadas. Finalmente, se suministran los mapas de distribución de cada especie, una gráfica con las curvas de fenología, así como una clave para su identificación.
\end{abstract}

Palabras clave: Ceratinini, Hymenoptera, Apoidea, Xylocopinae, área íbero-balear, islas Canarias, mapas de distribución, fenología, clave.

\begin{abstract}
New data on Spanish and Portuguese Ceratinini, with a key to their identification (Hymenoptera, Apoidea, Xylocopinae)

A review of the Iberian species of the tribe Ceratinini has been carried out, based upon data from several entomological collections and bibliographic antecedents. A total of 13 species of the genus Ceratina inhabit the Iberian Peninsula, the Balearic and Canary Islands. From our current knowledge, C. cucurbitina and C. cyanea are the most frequent species, whereas $C$. albosticta, new for this fauna, is the rarest, with just a single record; $C$. gravidula is also new for the Iberian fauna. C. acuta and C. loewi, recorded for the first time by Dusmet (1923), and almost certainly based on misidentifications, are removed from the Iberian list. Finally, distribution maps for every species, a graph showing phenology, and an identification key are provided.
\end{abstract}

Key words: Ceratinini, Hymenoptera, Apoidea, Xylocopinae, Ibero-balearic area, Canary Islands, distribution maps, phenology, key.

* Université de Mons-Hainaut, Laboratoire de Zoologie. Avenue du Champ de Mars. B-7000 Mons, Bélgica. Chargé de recherches FNRS. E-mail: michael.terzo@umh.ac.be

** Universidad de Almería, Grupo de Investigación "Transferencia de I+D en el Área de Recursos Naturales". E-04120 La Cañada de San Urbano (Almería), España. E-mail: fjortiz@ual.es 


\section{Introducción}

La tribu Ceratinini incluye a las abejas conocidas como "pequeñas carpinteras", nombre que deriva de sus hábitos de anidar en tallos de plantas, tales como zarzas y juncos. Son abejas esbeltas y de tamaño entre muy pequeño y mediano, con escasa pubescencia y tegumento negro o con brillo metálico verde o azulado, normalmente con manchas de color amarillo pálido o blanco marfil.

En Europa, este grupo está representado únicamente por el género Ceratina. Para la fauna española, Dusmet (1923) citó 12 especies, pero él mismo reiteraba la dificultad de identificar con seguridad a algunas de ellas, e incurrió en ciertos errores que condujeron a la cita de dos de ellas cuya presencia en nuestra fauna era muy dudosa $(C$. acuta y $C$. loewi). En cuanto a Portugal, el mismo Dusmet (1931) citaba 6 especies, incluyendo a C. acuta; Diniz, en revisiones posteriores, reiteró esas citas y añadió otras especies, con lo que el número de las publicadas en dicho territorio ascendió a 8 (ver, por ejemplo, Diniz, 1960). Finalmente, Daly (1983) publicó un extenso artículo, donde aportaba numerosos datos sobre taxonomía y ecología para toda la fauna del norte de África y la Península Ibérica. Ese trabajo establecía en 10 el número de especies ibéricas, pero no se pronunciaba sobre las publicadas por los autores arriba mencionados, trabajos que el autor desconocía o no tuvo en consideración.

\section{Material y métodos}

El material estudiado procede de los siguientes museos e instituciones:

Colecciones $\mathrm{N}^{\mathrm{o}}$ de ejemplares

Nationaal Natuurhistorisch Museum, Leiden (Países Bajos; Dr. C. van Achterberg) 743

Museo Nacional de Ciencias Naturales, Madrid (España; Dr. I. Izquierdo)

Faculté Universitaire des Sciences Agronomiques de Gembloux (Bélgica; Prof. C. Gaspar)

Université de Mons-Hainaut (Bélgica)

Natural History Museum, London (Gran Bretaña; M. T. Huddleston)

Muséum National d'Histoire Naturelle de Paris (Francia; Dr. J. Weulersse)

Departamento de Zoología de la Universidad de Coimbra (Portugal; Dr. M. de A. Diniz)

Oberösterreichisches Landesmuseums,

Linz (Austria; Mag. F. Gusenleitner)

Instituut voor Taxonomische Zöologie, Amsterdam (Países Bajos; Dr. W. Hogenes)

Museum für Naturkunde der Humbodt-Universität, Berlin (Alemania; Dr. F. Koch)

Naturhistorisches Museum, Wien (Austria; Dr. S. Schödl)

Zoologische Staatssammlung München (Alemania; Dr. E. Diller)

Institut Royal des Sciences Naturelles de Belgique, Bruxelles (Bélgica; Dr. P. Grootaert)

Muséum d'Histoire Naturelle de Strasbourg (Francia; M. J. Matter)

Tabla 1.- Número de ejemplares en las publicaciones que se han empleado para completar los mapas de distribución.

Table 1.- Number of specimens from publications used to complete the distribution maps.

\begin{tabular}{|c|c|c|c|c|c|}
\hline & Daly, 1983 & Dusmet, 1923 & Espeso \& Gayubo, 1988 & Gayubo et al., 1989 & Heras \& Gayubo, 1989 \\
\hline C. callosa & 276 & & 4 & 1 & \\
\hline C. chalcites & 67 & & 1 & & 10 \\
\hline C. chalybea & 52 & & 1 & 6 & 1 \\
\hline C. cucurbitina & 116 & & 9 & 52 & 15 \\
\hline C. cyanea & 427 & & 2 & 9 & 3 \\
\hline C. dallatorreana & 12 & & 1 & & 1 \\
\hline C. dentiventris & 5 & & 1 & 21 & \\
\hline C. mocsaryi & 4 & & & 1 & \\
\hline C. nigrolabiata & 269 & & 1 & 1 & \\
\hline C. parvula & & 5 & & & \\
\hline C. saundersi & 1 & & & & \\
\hline
\end{tabular}

* incluyendo 9 ejemplares identificados como C. acuta / including 9 specimens identified as C. acuta.

** incluyendo 1 macho identificado como $C$. dallatorreana / including 1 male identified as $C$. dallatorreana. 
Zoological Institut of Russian Academy of Sciences, Saint Petersburg (Rusia; Dr. Yu. A. Pesenko)

Deutsches Entomologisches Institut, Eberswalde (Alemania; Prof. H. Dathe)

TOTAL

y de las siguientes colecciones privadas:

\section{Colecciones}

F. J. Ortiz-Sánchez (España)

J. Leclercq (Bélgica)

R. Wahis (Bélgica)

C. Schmid-Egger (Alemania)

G. van der Zanden (Países Bajos) $\dagger$

J. Maldes (Francia)

M. Kuhlmann (Alemania)

P. Westrich (Alemania)

A. Pauly (Bélgica)

G. Le Goff (Francia)

R. Constantin (Francia)

A. W. Ebmer (Austria)

F. Burger (Alemania)

F. Amiet (Francia)

TOTAL

$\mathrm{N}^{0}$ de ejemplares

TOTAL instituciones + colecciones privadas

MAPAS DE DISTRIBUCIÓN: Los mapas de distribución han sido dibujados mediante el programa informático Carto Fauna-Flora 2.0, (Barbier \& Rasmont, 2000). Tipo de proyección: cónica, centrada en latitud $3^{\circ} \mathrm{W}$. Diámetro de los puntos: 30 km. Para completar los mapas de distribución se han añadido todos o parte de los datos publicados en algunos artículos científicos, según se muestra en la Tabla 1.

Terminología empleadA: Para la mejor interpretación de la clave de identificación que se incluye más abajo, indicamos a continuación algunos caracteres con los que el no especialista puede no estar familiarizado.

En la cabeza, la carena hipostomal tiene un alto valor taxonómico. Se trata de una quilla más o menos fuertemente desarrollada, situada en la parte posterior de la cabeza, que corre a los lados de la cavidad oral y la delimita de la mejilla y el occipucio. El área supra-antenal se extiende entre los ocelos y las fosetas antenales, el área paraocular está delimitada por los ojos compuestos y, hacia el interior, por el clípeo y las fosetas antenales y, finalmente, el área paraocelar es aquélla que queda adyacente a los ocelos.

En el "tórax" (resultado de la fusión del tórax verdadero con el primer segmento abdominal, o propodeo), el pronoto se extiende posterolateralmente, dando lugar a los lóbulos pronotales (= callos humerales). El mesoscutum (= escudo) es el principal esclerito del mesonoto.

En el gáster, se designa abreviadamente a terguitos y esternitos por su inicial, es decir, $\mathrm{T}$ y $\mathrm{E}$, respectivamente, seguida de un subíndice que

\begin{tabular}{|c|c|c|c|c|c|}
\hline Ornosa, 1993 & Ornosa \& Martínez, 1996 & Pérez-Íñigo, 1982 & Pérez-Íñigo \& Gayubo, 1989 & Saunders, 1901 & Torres et al., 1989 \\
\hline 1 & & 24 & & & $\begin{array}{l}1 \\
3\end{array}$ \\
\hline 27 & $\begin{array}{l}2 \\
3\end{array}$ & $\begin{array}{l}147 \\
23^{*}\end{array}$ & $\begin{array}{c}100 \\
2\end{array}$ & 1 & $\begin{array}{l}2 \\
2 \\
\end{array}$ \\
\hline $\begin{array}{l}2 \\
9 \\
4 \\
\end{array}$ & $\begin{array}{l}4 \\
2\end{array}$ & $\begin{array}{c}10 \\
1 \\
4 \\
\end{array}$ & $\begin{array}{l}9 * * \\
1 \\
1 \\
\end{array}$ & & $\begin{array}{l}9 \\
1 \\
1 \\
\end{array}$ \\
\hline & & & & 1 & \\
\hline
\end{tabular}


Tabla 2.- Número total de ejemplares tomados en consideración (ejemplares estudiados + datos publicados mencionados en la Tabla 1).

Table 2.- Total number of specimens considered (specimens studied + published records, mentioned in Table 1). See Table 3 for the detail of the Peninsula.

\begin{tabular}{|c|c|c|c|c|c|c|c|}
\hline \multirow[t]{2}{*}{ Género Ceratina Latreille, 1802} & \multirow[t]{2}{*}{ Portugal } & \multicolumn{5}{|c|}{ España (ver Tabla 3 para el detalle de la Península) } & \multirow[t]{2}{*}{ Total } \\
\hline & & Península & Mallorca & Menorca & Melilla & Gran Canaria & \\
\hline \multicolumn{8}{|l|}{ Subgénero Ceratina Latreille $s$. str. } \\
\hline Ceratina cucurbitina (Rossi, 1792) & 95 & 808 & 13 & 2 & & & 918 \\
\hline Ceratina parvula Smith, 1854 & 5 & 16 & 3 & & & 2 & 26 \\
\hline \multicolumn{8}{|c|}{ Subgénero Euceratina Hirashima, Moure \& Daly, 1971} \\
\hline Ceratina albosticta Cockerell, 1931 & & 1 & & & & & 1 \\
\hline Ceratina callosa (Fabricius, 1794) & 9 & 378 & & & & & 387 \\
\hline Ceratina chalcites Germar, 1839 & 5 & 216 & & & & & 221 \\
\hline Ceratina chalybea Chevrier, 1872 & 17 & 184 & & & & & 201 \\
\hline Ceratina cyanea (Kirby, 1802) & 87 & 792 & & & & & 879 \\
\hline Ceratina dallatorreana Friese, 1896 & 8 & 145 & 11 & 2 & 1 & & 167 \\
\hline Ceratina dentiventris Gerstaecker, 1869 & 3 & 85 & & & & & 88 \\
\hline Ceratina gravidula Gerstaecker, 1869 & 1 & 2 & & & & & 3 \\
\hline Ceratina mocsaryi Friese, 1896 & 13 & 46 & & & & & 59 \\
\hline Ceratina nigrolabiata Friese, 1896 & 14 & 347 & & & & & 361 \\
\hline Ceratina saundersi Daly, 1983 & 17 & 44 & & & & & 61 \\
\hline TOTAL & 274 & 3.064 & 27 & 4 & 1 & 2 & 3.372 \\
\hline
\end{tabular}

corresponde al ordinal del segmento gastral referido. Con ello, por ejemplo, el tercer terguito se abrevia como $\mathrm{T}_{3}$ y el quinto esternito como $\mathrm{E}_{5}$. En las hembras, la base de $\mathrm{E}_{2}$ y $\mathrm{E}_{3}$ presenta, frecuentemente, sendas áreas más o menos semilunares, las glándulas de la cera.

\section{Resultados}

Tras el estudio de los ejemplares reseñados, el catálogo de las especies de Ceratinini de España y Portugal contiene 13 especies ${ }^{1}$ (Tabla 2). Se ha empleado el encuadre subgénerico tradicionalmente reconocido (por ejemplo, Daly, 1983), rechazando, por tanto, la posible inclusión de C. parvula Smith, 1854 en el subgénero Ceratinula Moure, 1941, planteada por Michener (2000).

La distribución actualmente conocida de cada una de estas especies está resumida en las Tablas 2 y 3 y representada en los mapas que aparecen en las figuras 33-46. El número total de ejemplares estudiados (incluyendo los datos publicados), 4.129 , es mayor que el que aparece en los mapas,

\footnotetext{
${ }^{1}$ La lista sinonímica puede encontrarse en Daly (1983) y Terzo \& Rasmont (1996).
}

3.699. La diferencia (430 ejemplares) se debe a los registros que ha sido imposible localizar adecuadamente.

\section{Clave para las especies ibéricas del género Ceratina $^{2}$}

1. Tegumento negro, sin brillo metálico; áreas supra-antenal y paraocular y mesoscutum con amplias zonas lisas, sin escultura ............ (subgénero Ceratina Latreille, 1802) 2

- Tegumento con reflejos metálicos de color azul o verde; áreas supra-antenal y paraocular y mesoscutum con punteado denso (subgénero Euceratina Hirashima, Moure \& Daly, 1971) 3

2. Longitud del ala anterior mayor que $4 \mathrm{~mm}$; cara con algún punteado; mejillas redondeadas; coxas de las patas delanteras simples ..................... C. cucurbitina (Rossi, 1792)

- Longitud del ala anterior menor que $3 \mathrm{~mm}$; cara sin punteado; mejillas angulosas en visión lateral; ángulo laterobasal de las coxas de las patas delanteras extendido lateralmente .................................. C. parvula Smith, 1854

3. Longitud del ala anterior mayor que $7 \mathrm{~mm}$; $\mathrm{E}_{2}$ del macho con un diente central que puede ser tan largo como la longitud del esternito .................. C. chalcites Germar, 1839

- Longitud del ala anterior menor que $7 \mathrm{~mm}$; $\mathrm{E}_{2}$ del macho plano o con un diente muy pequeño $(C$. dentiventris $)$.... 4

4. Machos: antenas con escapo, pedicelo y 11 flagelómeros; gáster con 7 terguitos visibles

... 5 


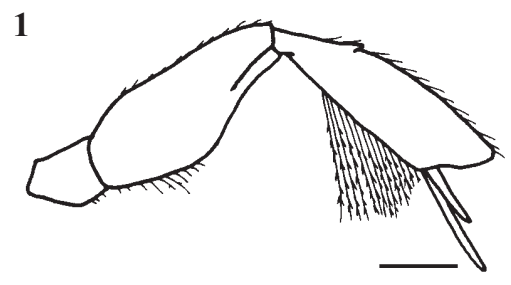

2
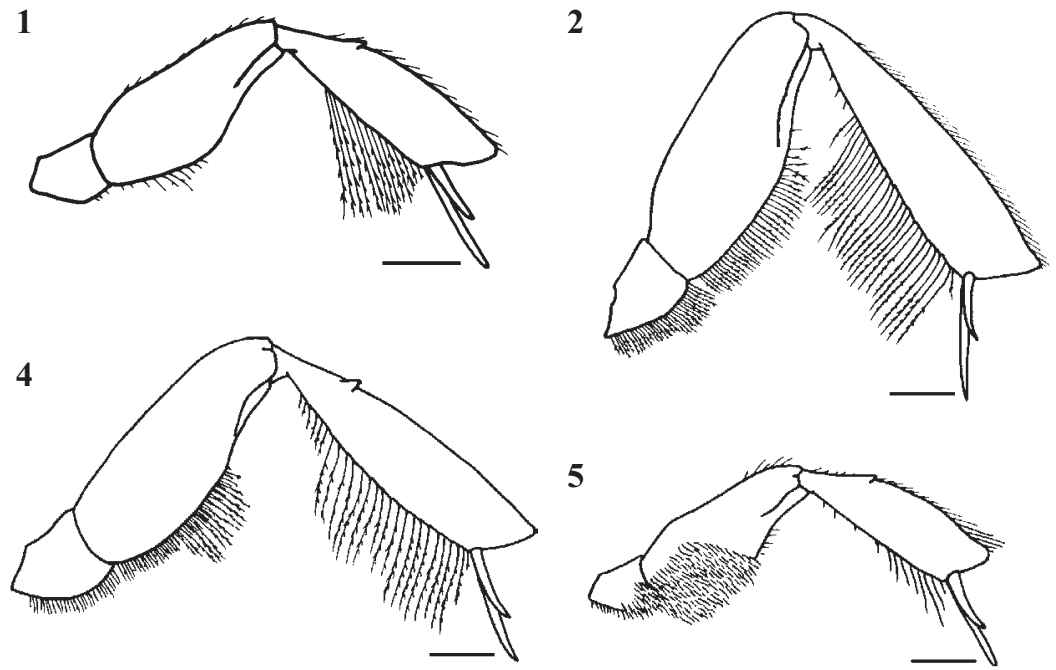

7

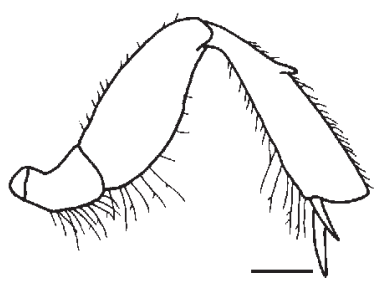

10

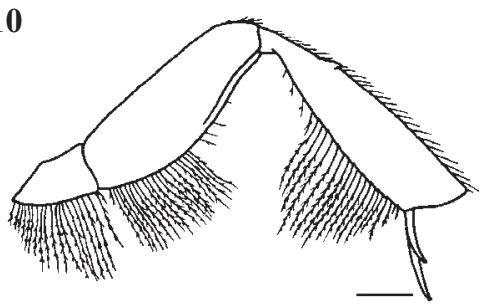

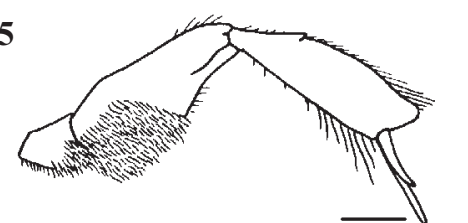

8

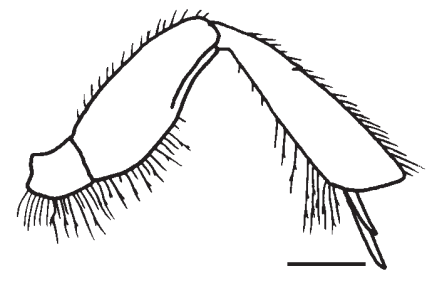

11

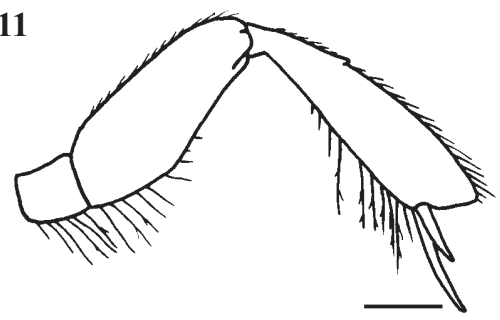

3

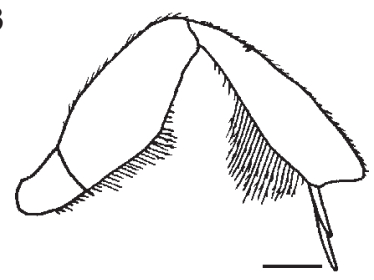

6

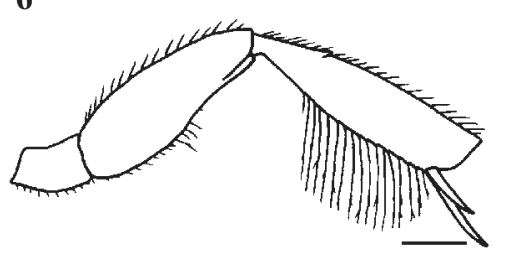

9

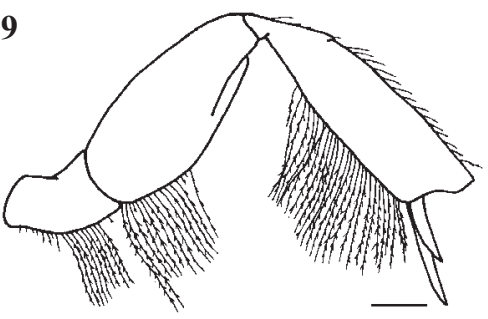

12

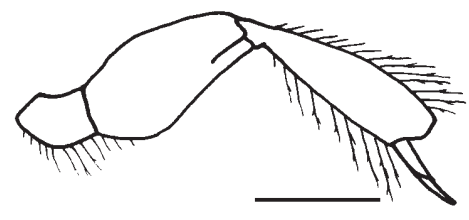

13

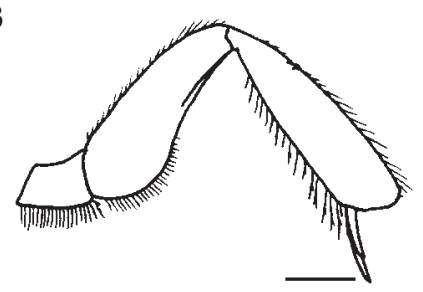

Figs. 1-13.- Pata posterior izquierda del macho de: 1) Ceratina albosticta; 2) C. callosa; 3) C. chalcites; 4) C. chalybea; 5) C. cucurbitina; 6) C. cyanea; 7) C. dallatorreana; 8) C. dentiventris; 9) C. gravidula; 10) C. mocsaryi; 11) C. nigrolabiata; 12) C. parvula; 13) C. saundersi. Escala: 1, 2, 4-13 =0,25 mm; $3=0,50 \mathrm{~mm}$.

Figs. 1-13.- Male left hind leg. 1) Ceratina albosticta; 2) C. callosa; 3) C. chalcites; 4) C. chalybea; 5) C. cucurbitina; 6) C. cyanea; 7) C. dallatorreana; 8) C. dentiventris; 9) C. gravidula; 10) C. mocsaryi; 11) C. nigrolabiata; 12) C. parvula; 13) C. saundersi. Scale: $1,2,4-13=0.25 \mathrm{~mm} ; 3=0.50 \mathrm{~mm}$. 
14

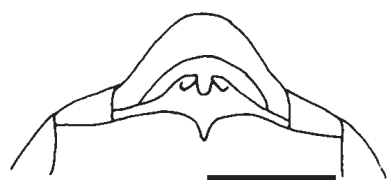

17

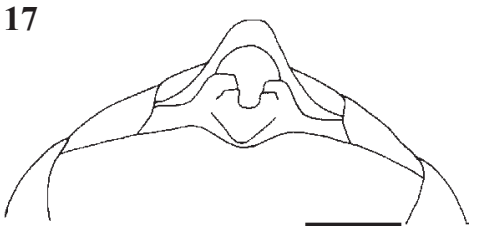

20

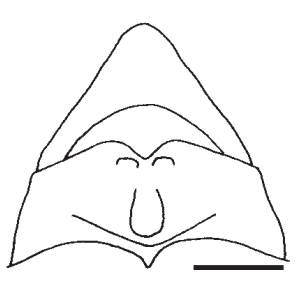

23

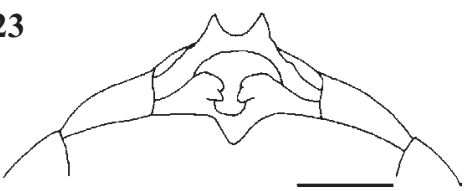

15

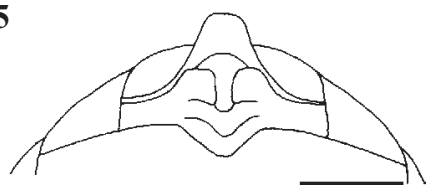

18

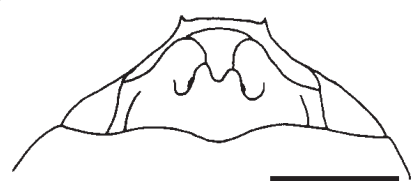

21

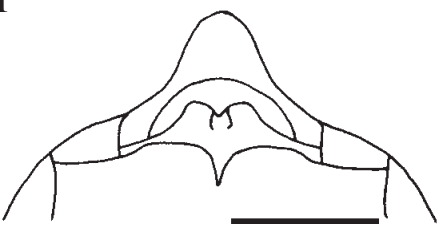

24

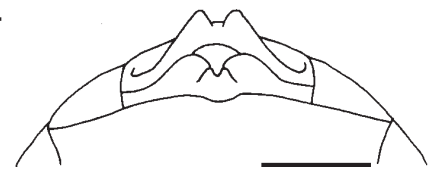

16

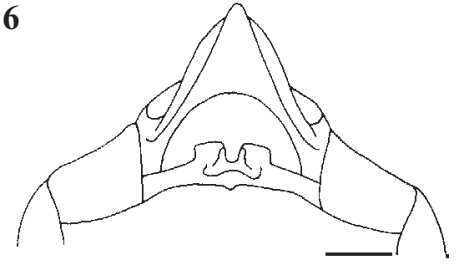

19

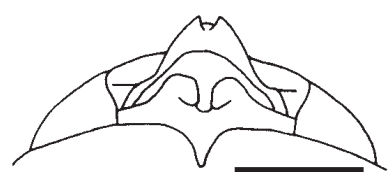

22

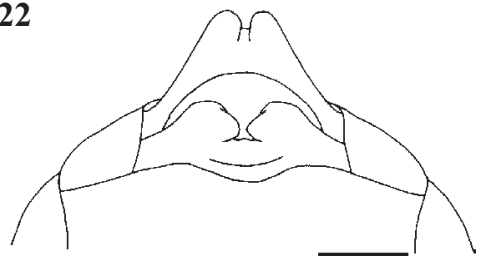

25

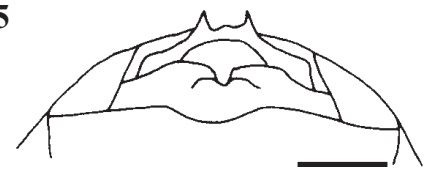

26

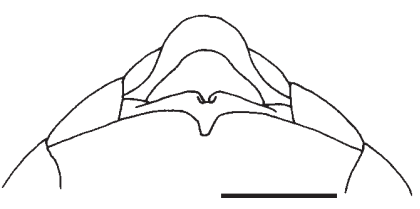

27

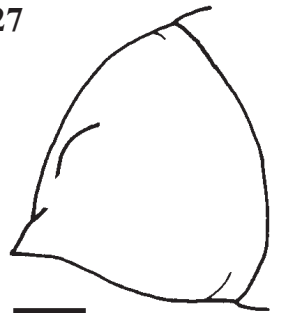

28

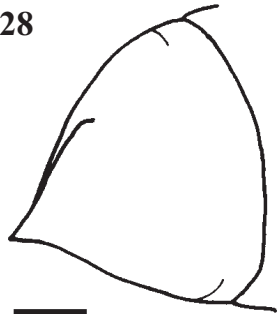

Figs. 14-28.- Últimos terguitos y esternitos, en vista ventral, del macho de: 14) Ceratina albosticta; 15) C. callosa; 16) C. chalcites; 17) C. chalybea; 18) C. cucurbitina; 19) C. cyanea; 20) C. dallatorreana; 21) C. dentiventris; 22) C. gravidula; 23) C. mocsaryi; 24) C. nigrolabiata; 25) C. parvula; 26) C. saundersi. $\mathrm{E}_{6}$, en vista lateral, de la hembra de: 27) Ceratina albosticta y 28) $C$. saundersi. Escala: $14-19,21-24,26=0,50 \mathrm{~mm} ; 20,25=0,25 \mathrm{~mm} ; 27-28=0,25 \mathrm{~mm}$.

Figs. 14-28.- Male last terga and sterna in ventral view. 14) Ceratina albosticta; 15) C. callosa; 16) C. chalcites; 17) C. chalybea; 18) C. cucurbitina; 19) C. cyanea; 20) C. dallatorreana; 21) C. dentiventris; 22) C. gravidula; 23) C. mocsaryi; 24) C. nigrolabiata; 25) C. parvula; 26) C. saundersi. Scale: 14-19, 21-24, $26=0.50 \mathrm{~mm} ; 20,25=0.25 \mathrm{~mm}$. Female $\mathrm{S}_{6}$ in lateral view. 27 , Ceratina albosticta; 28, C. saundersi. Scale $=0.25 \mathrm{~mm}$. 


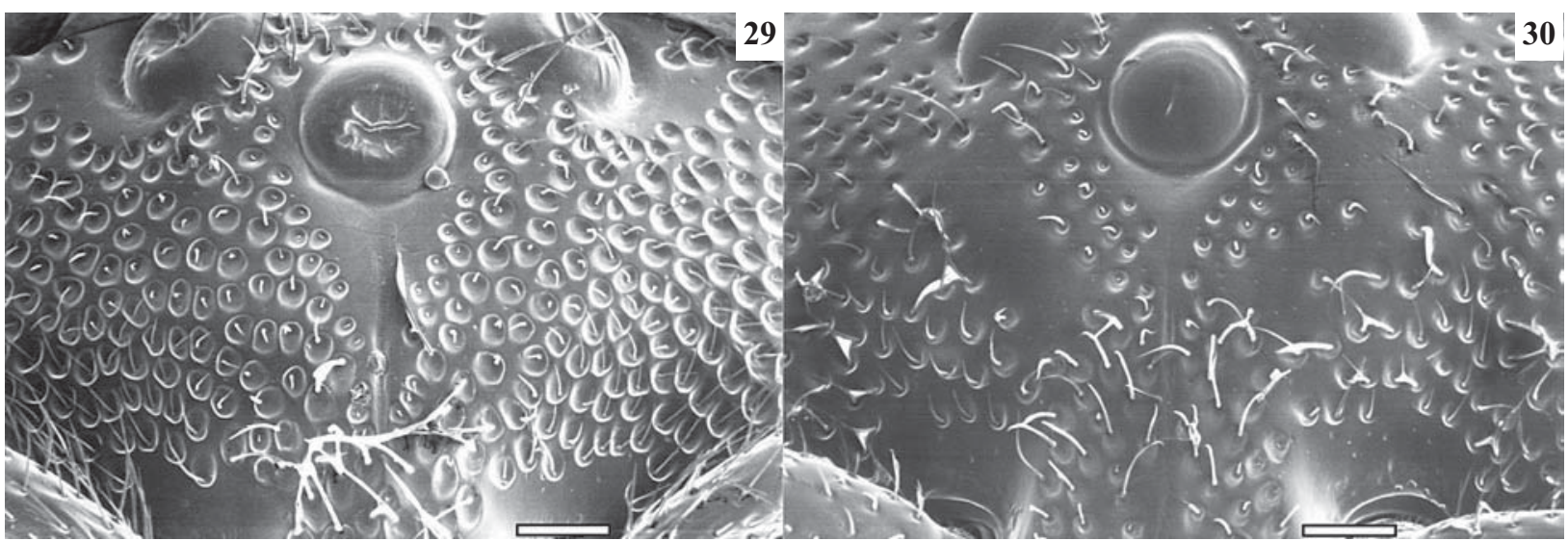

Figs. 29-30.- Área ocelar de la hembra de: 29) Ceratina cyanea y 30) C. nigrolabiata. Escala $=100 \mu \mathrm{m}$.

Figs. 29-30.- Female ocellar area. 29) Ceratina cyanea; 30) C. nigrolabiata. Scale $=100 \mu \mathrm{m}$.

- Hembras: antenas con escapo, pedicelo y 10 flagelómeros; gáster con 6 terguitos visibles ...................................... 14

5. Extremo de $\mathrm{T}_{7}$ bidentado o bilobulado (figs. 19, 22-24)

- Extremo de $\mathrm{T}_{7}$ apuntado, redondeado, algunas veces truncado, pero nunca emarginado (figs. 14-16, 20, 21, 26)

6. Labro, lóbulos pronotales $\mathrm{y}_{4} \mathrm{a}_{6}$ negros, sin brillo metálico; mancha clipeal reducida a un pequeño triángulo (si es mayor, nunca alcanza los márgenes laterales ni el apical); patas traseras y extremo del gáster según las figuras 11 y

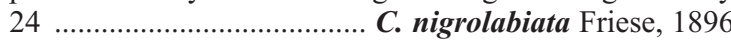

- Labro y/o lóbulos pronotales generalmente con una mancha de color marfil, $\mathrm{T}_{4}$ a $\mathrm{T}_{6}$ con brillo metálico; la mancha del clípeo ocupa casi toda su extensión

7. Pubescencia ventral del fémur de las patas traseras más corta que la anchura máxima del fémur (fig. 6); extremo del gáster como en la figura 19 ........ C. cyanea (Kirby, 1802)

- Pubescencia ventral del fémur de las patas traseras más larga que la anchura máxima del fémur ......................... 8

8. Carena hipostomal fuertemente desarrollada y convexa; extremo de $\mathrm{T}_{7}$ bidentado (fig. 23); cara ventral del tórax y primeros esternitos gastrales con pubescencia inusualmente larga y densa de color blanco; patas traseras como en la figura 10 ......................... C. mocsaryi Friese, 1896

- Carena hipostomal débil y ligeramente cóncava; extremo de $\mathrm{T}_{7}$ bilobulado (fig. 22); cara ventral del tórax y primeros esternitos con pubescencia corta y dispersa; patas traseras como en la figura 9 .......... C. gravidula Gerstaecker, 1869

9. Pubescencia ventral de la tibia de las patas traseras más corta que la anchura máxima de la tibia (figs. $7,8,13$ ) .......... 10

- Pubescencia ventral de la tibia de las patas traseras más larga que la anchura máxima de la tibia (figs. $1,2,4) \quad . . . \ldots \ldots . . . .12$

10. Mancha del clípeo con punteado; palpos maxilares con 5 subdivisiones; extremo de las coxas de las patas traseras con un diente apical romo en su cara interna; patas traseras y extremo del gáster como en las figuras 13 y $26 \ldots . .$.

C. saundersi Daly, 1983
- Mancha del clípeo sin punteado, lisa; palpos maxilares con 6 subdivisiones; coxas de las patas traseras sin diente apical

11. $E_{2}$ con un pequeño diente en el centro; $T_{6}$ con una carena media débilmente indicada o una línea sin punteado; patas traseras y extremo del gáster como en las figuras 8 y 21

C. dentiventris Gerstaecker, 1869

- $\quad \mathrm{E}_{2} \sin$ diente; $\mathrm{T}_{6}$ sin carena media ni línea sin punteado; patas traseras y extremo del gáster como en las figuras 7 y 20 .................................. C. dallatorreana Friese, 1896

12. Longitud del ala anterior menor que $5 \mathrm{~mm}$; palpos maxilares con 5 subdivisiones; patas traseras y extremo del gáster como en las figuras 1 y 14 .. C. albosticta Cockerell, 1931

- Longitud del ala anterior mayor que $5 \mathrm{~mm}$; palpos maxilares con 6 subdivisiones

13. Extremo de $\mathrm{T}_{7}$ ampliamente redondeado, su borde lateral es convexo (fig. 17); pubescencia ventral del fémur de las patas traseras: un cepillo flojo de setas que se alarga en el extremo (fig. 4); carena hipostomal débil detrás de las mandíbulas, pero abruptamente más desarrollada cerca del occipucio …………............... C. chalybea Chevrier, 1872

- Extremo de $\mathrm{T}_{7}$ más largo, estrechado, redondeado sólo en el ápice y con borde lateral recto o cóncavo (fig. 15); pubescencia ventral del fémur de las patas traseras: un cepillo corto y denso de longitud uniforme (fig. 2); carena hipostomal uniformemente débil en toda su longitud ......

C. callosa (Fabricius, 1794)

14. Área paraocelar con punteado denso: junto al ocelo medio carece de una superficie lisa sin punteado o dispersamente punteada (fig. 29); carena hipostomal diferente; lóbulos pronotales negros o blancos; $\mathrm{T}_{4}$ a $\mathrm{T}_{6}$ con brillo metálico

- Área paraocelar con punteado más disperso: los espacios entre los puntos y entre éstos y el ocelo medio son mayores o iguales que el diámetro de los puntos (fig. 30); carena hipostomal recta, uniformemente desarrollada y, en cada extremo, en ángulo recto; lóbulos pronotales y $\mathrm{T}_{4}$ a $\mathrm{T}_{6}$ negros sin brillo metálico .... C. nigrolabiata Friese, 1896 


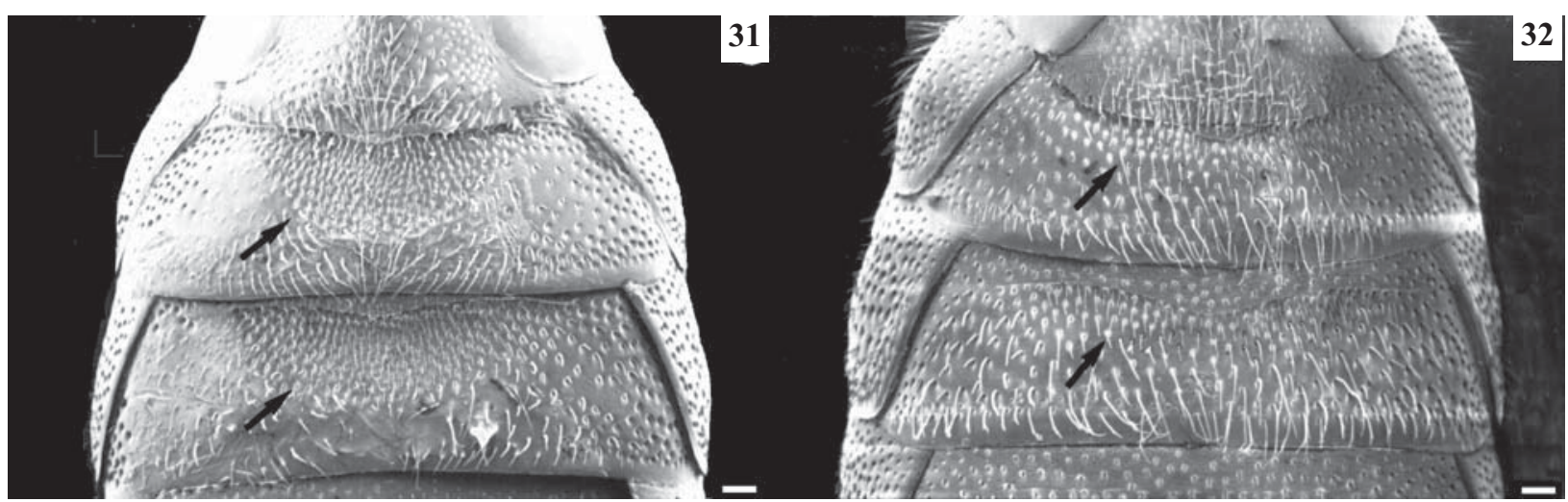

Figs. 31-32.- Glándulas de la cera (flecha negra) de $\mathrm{E}_{2}$ y $\mathrm{E}_{3}$ en la hembra de: 31, Ceratina dallatorreana y 32, C. cyanea. Escala $=100 \mu \mathrm{m}$.

Figs. 31-32.- Female wax glands (black arrow) on $\mathrm{S}_{2}$ and $\mathrm{S}_{3} .31$, Ceratina dallatorreana; 32, C. cyanea. Scale $=100 \mu \mathrm{m}$.

15. Especies grandes: longitud del ala anterior mayor que $5 \mathrm{~mm}$; carena hipostomal débil a fuertemente desarrollada ........ 16

- Especies pequeñas: longitud del ala anterior menor que $5 \mathrm{~mm}$; carena hipostomal siempre débilmente desarro-

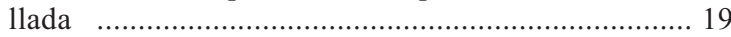

16. Mancha clipeal pequeña o ausente, pero situada sobre una franja carente de punteado en el centro del clípeo ....... 17

- Mancha clipeal grande o pequeña, pero el centro del clípeo siempre punteado, carente de una franja media lisa .... 18

17. Carena hipostomal fuertemente desarrollada, curvada mancha clipeal pequeña o ausente; lóbulos pronotales siempre de color marfil ............ C. mocsaryi Friese, 1896

- Carena hipostomal débil; mancha clipeal en forma de franja larga y estrecha, raras veces ausente; lóbulos pronotales negros ............... C. gravidula Gerstaecker, 1869

18. Mitad posterior de la carena hipostomal abruptamente desarrollada; mancha clipeal grande y de forma oval, raras veces reducida C. chalybea Chevrier, 1872

- Carena hipostomal débil; mancha clipeal ausente o muy pequeña ............................... C. callosa (Fabricius, 1794)

19. La mancha basitibial del par de patas anterior se extiende, al menos, hasta la mitad de la tibia; mancha clipeal bien desarrollada y a menudo amplia; lóbulos pronotales siempre de color marfil; tegumento verdoso; glándulas de la cera bien definidas, ambas de forma semilunar y siempre tan largas como anchas (fig. 31) ......... C. dallatorreana Friese, 1896

- La mancha basitibial del par de patas anterior se reduce al tercio proximal de la tibia; mancha clipeal bien desarrollada o no; lóbulos pronotales negros o de color marfil; tegumento azul, raras veces verdoso; glándulas de la cera no bien definidas, ambas mucho más anchas que largas (fig. 32) (excepto en C. dentiventris) .......................... 20

20. Palpos maxilares con 5 subdivisiones; lóbulos pronotales siempre de color marfil

.. 21

- Palpos maxilares con 6 subdivisiones; lóbulos pronotales negros o de color marfil 22

21. $T_{6}$ con una carena media de perfil sinuoso (fig. 27); lóbulos pronotales con un punto apical de color marfil; mancha clipeal casi redonda C. albosticta Cockerell, 1931
- $\quad \mathrm{T}_{6}$ con una carena media de perfil casi recto (fig. 28); lóbulos pronotales de color marfil; mancha clipeal alargada

C. saundersi Daly, 1983

22. Longitud del ala anterior menor que $4 \mathrm{~mm}$; clípeo muy escasamente punteado con, al menos, una franja central lisa y una estrecha mancha longitudinal de color marfil; lóbulos pronotales siempre de color marfil; glándulas de la cera bien definidas, semilunares (fig. 31)

\section{C. dentiventris Gerstaecker, 1869}

- Longitud del ala anterior mayor que $4 \mathrm{~mm}$; clípeo densamente punteado, sin franja central lisa; mancha clipeal ausente o muy pequeña; lóbulos pronotales negros o de color marfil; glándulas de la cera poco definidas, ambas mucho más anchas que largas (fig. 32)

C. cyanea (Kirby, 1802)

Fenología: La figura 47 muestra la fenología del género Ceratina. Se representan, en conjunto, todas las especies presentes en España y Portugal. Cada una de ellas, individualmente, muestra curvas similares, lo que significa que, en cada especie, los machos aparecen antes que las hembras, en marzo y abril. Los machos son más abundantes en junio, mientras que las hembras lo son en julio. Machos y hembras desaparecen casi completamente en octubre. Las escasas capturas de noviembre se deben a menudo a individuos extraídos de los nidos durante la hibernación.

\section{Discusión}

Dos especies, C. albosticta y C. gravidula, se añaden aquí desde la última revisión de las especies 
Tabla 3.- Resumen de la distribución de las especies en las provincias de España peninsular ("X" indica la presencia de la especie; "+" indica la presencia de la especie según Ceballos, 1956, pero no confirmada; "?" indica la presencia de la especie registrada por Espeso \& Gayubo, 1988, pero para la cual no hay datos publicados u originales).

Table 3. Summary of the distribution of species within the continental Spanish provinces ("X" indicates the presence of the species; "+" indicates the presence of the species after Ceballos, 1956, but not confirmed; "?" indicates the presence of the species recorded by Espeso \& Gayubo, 1988 but for which there is no published or original data).

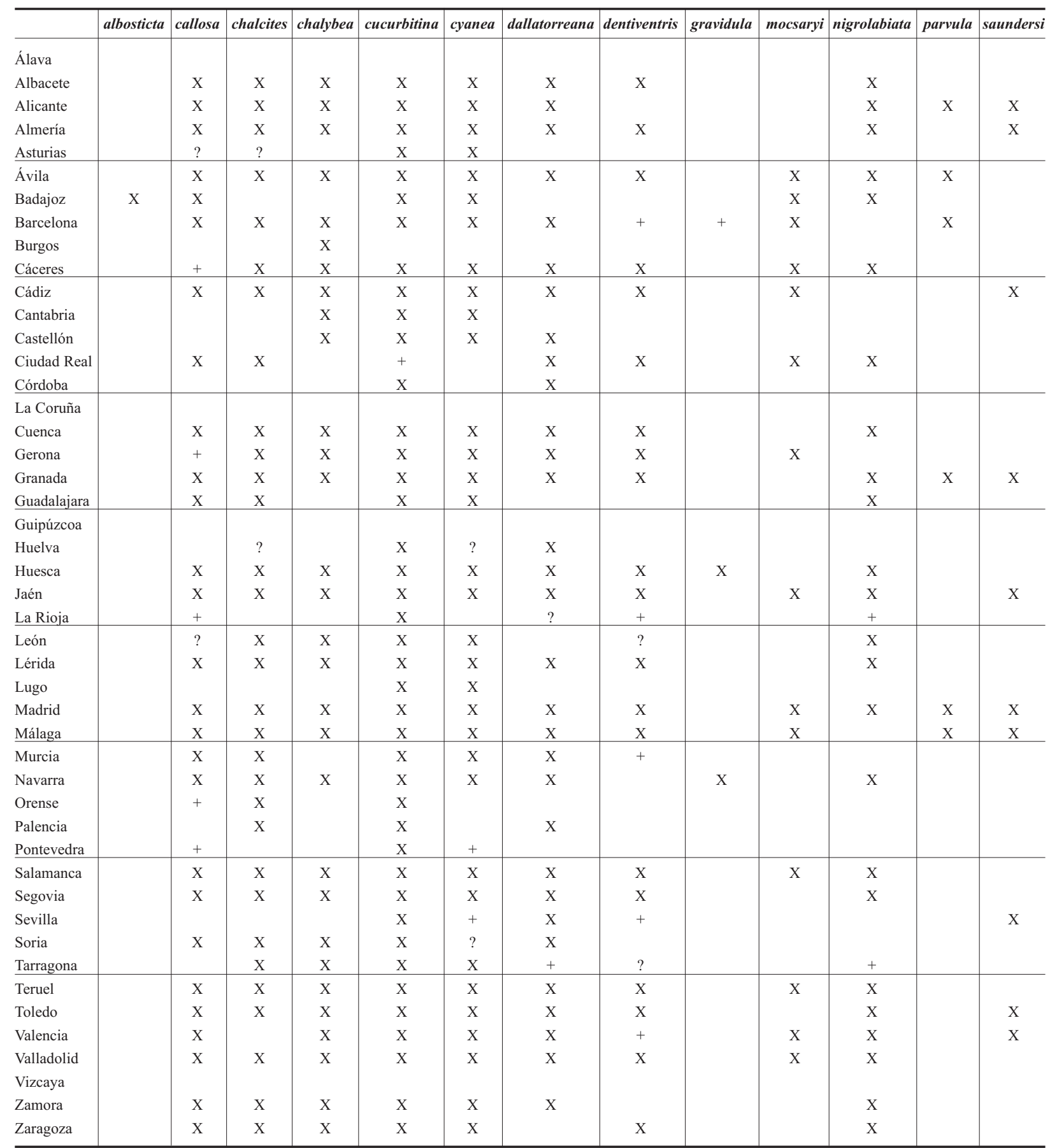



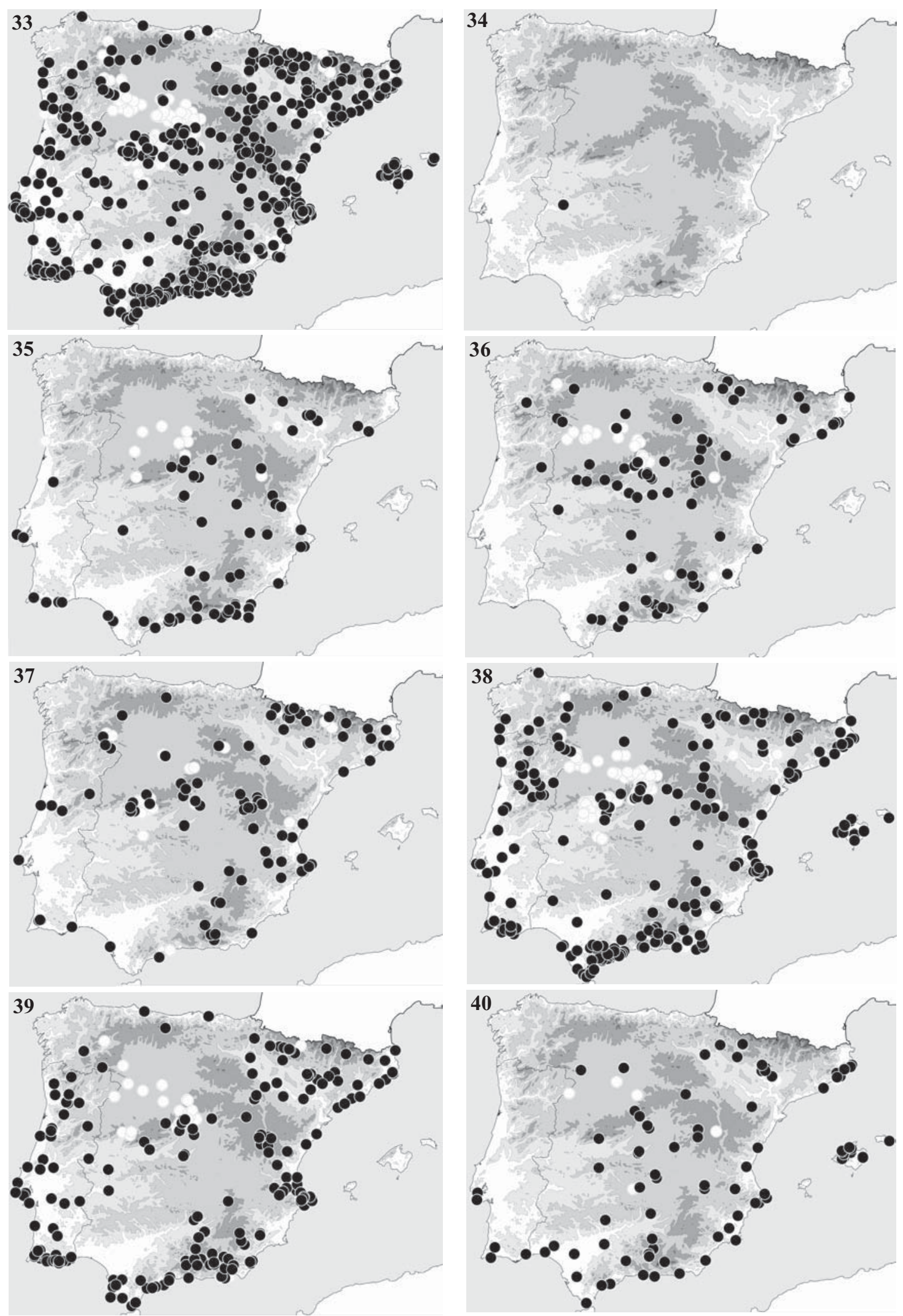

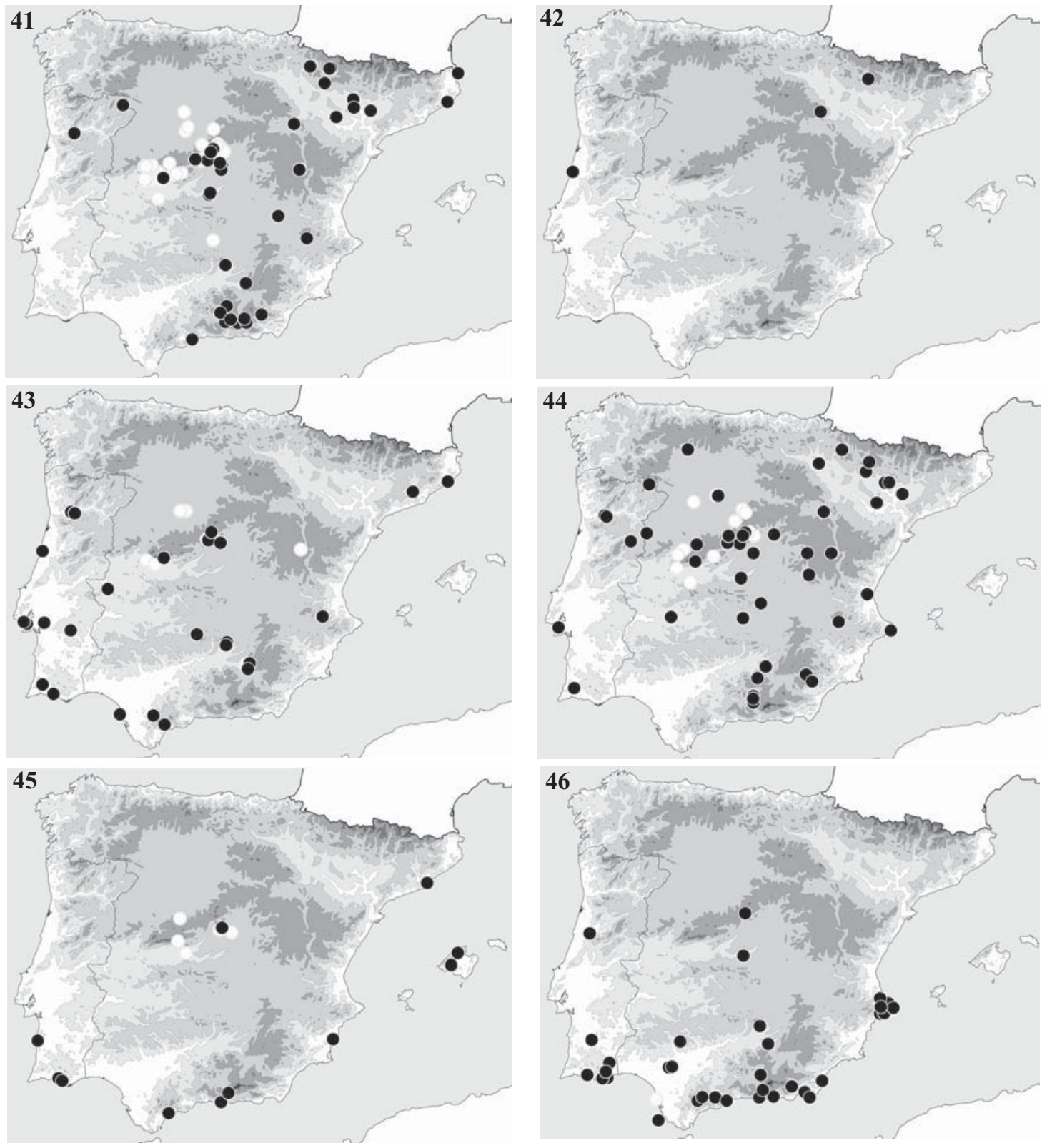

Figs. 33-46.- Mapas de distribución de las especies de Ceratinini ibéricas (círculos blancos: datos de la bibliografía; círculos negros: datos originales): 33 , todas las especies (1.761 ejemplares tomados de la bibliografía +1.938 datos originales); 34 , C. albosticta ( 1 dato original); 35 , C. callosa (282 ejemplares de la bibliografía +110 datos originales); 36, C. chalcites (113 ejemplares de la bibliografía + 127 datos originales); 37, C. chalybea (70 ejemplares de la bibliografía +146 datos originales; 38, C. cucurbitina (452 ejemplares de la bibliografía +705 datos originales); 39, C. cyanea (461 ejemplares de la bibliografía +407 datos originales); 40, C. dallatorreana (17 ejemplares de la bibliografía +148 datos originales); 41, C. dentiventris (60 ejemplares de la bibliografía +68 datos originales); $42, C$. gravidula (3 datos originales); 43, C. mocsaryi (18 ejemplares de la bibliografía + 62 datos originales); 44, C. nigrolabiata (281 ejemplares de la bibliografía +88 datos originales); 45 , C. parvula (6 ejemplares de la bibliografía +18 datos originales); 46, C. saundersi (1 ejemplar de la bibliografía +54 datos originales).

Figs. 33-46.- Distribution maps of Iberian Ceratinini species (white dots: data from literature; black dots: original data): 33, all species (1,761 specimens from literature $+1,938$ original data); 34 , C. albosticta (1 original data); 35 , C. callosa ( 282 spec. from literature +110 original data); 36, C. chalcites (113 spec. from literature +127 original data); 37, C. chalybea $(70$ spec. from literature +146 original data); 38, C. cucurbitina (452 spec. from literature +705 original data); 39 , C. cyanea $(461 \mathrm{spec}$. from literature +407 original data); 40 , C. dallatorreana (17 spec. from literature +148 original data); 41 , C. dentiventris ( 60 spec. from literature +68 original data); $42, C$. gravidula (3 original data); 43, C. mocsaryi (18 spec. from literature +62 original data); 44, C. nigrolabiata (281 spec. from literature + 88 original data); 45, C. parvula (6 spec. from literature +18 original data); 46, C. saundersi ( $1 \mathrm{spec}$. from literature +54 original data). 


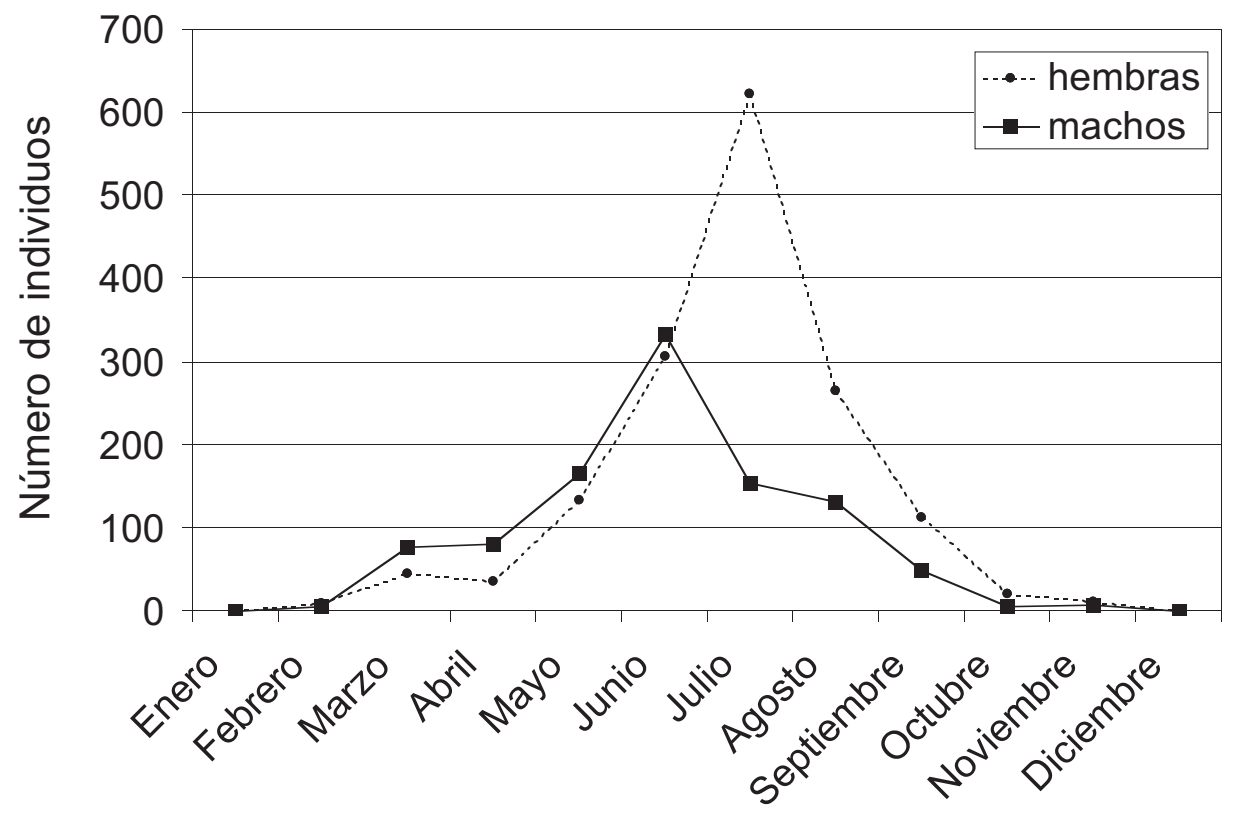

Fig. 47.- Curvas de fenología del género Ceratina en el área íbero-balear.

Fig. 47.- Phenology charts of the genus Ceratina in the Ibero-balearic area.

ibéricas de Ceratina (Daly, 1983). Por el contrario, C. acuta y $C$. loewi, dos especies comúnmente registradas en España por varios autores (Dusmet, 1923; Pérez-Iñigo，1980，1982; Pérez-Iñigo \& Gayubo, 1989), son eliminadas del catálogo ibérico de Dusmet (1923) y Ceballos (1956). No se ha encontrado ningún ejemplar que se pueda adjudicar a ninguna de ellas; sus citas se deben seguramente a identificaciones incorrectas ${ }^{3}$. En efecto, ambas se presentan en países del Mediterráneo oriental (Terzo, 1998; Terzo \& Rasmont, 1998). Es fácil confundir a C. acuta con algunos ejemplares españoles de $C$. cyanea con lóbulos pronotales blancos, mientras que $C$. loewi puede ser confundida con $C$. chalybea o C. callosa cuando se emplea la clave de Friese (1896) para las especies paleárticas.

Según Daly (1983), C. saundersi es una especie común en el norte de África. Este autor citaba sólo un ejemplar de España (Cádiz: Rota, UTM: 29SQA3661); sin embargo, el mapa de la figura 46 muestra cómo esta especie está muy ampliamente

3 El material estudiado por Dusmet, y depositado en el Museo Nacional de Ciencias Naturales de Madrid, ha sido revisado recientemente (datos en vías de publicación) y su examen confirma que se trata de ejemplares identificados incorrectamente. representada en el sur de la Península. Por el contrario, C. maghrebensis Daly, 1983, cuya distribución conocida es también el norte de África y cuya presencia en el sur de España es probable, según Terzo \& Rasmont (1993), no ha podido ser encontrada a partir del material estudiado hasta ahora, pero no se descarta que en el futuro pueda encontrarse en la Península.

Ceratina gravidula ya había sido citada en España por Dusmet (1923), concretamente en la provincia de Barcelona; sin embargo, con el material ahora estudiado no se puede confirmar su presencia en esa provincia. Por otra parte, se cita ahora esta especie por primera vez de Portugal (1 hembra, Figueira da Foz, Coimbra, 4012'10'N 847'44”'W, 5-VIII-1968, M. A. Diniz leg., Departamento de Zoología de la Universidad de Coimbra), en una localidad bastante alejada del registro más próximo en España (ver fig. 42).

Sólo existen datos de una especie en Melilla; se trata de una hembra de $C$. dallatorreana. No obstante, se conocen 15 especies en Marruecos (Daly, 1983; Terzo \& Rasmont, 1998).

Parece que sólo existen tres especies en las Islas Baleares: C. cucurbitina, $C$. dallatorreana y $C$. parvula; aparecen principalmente en Mallorca, 
aunque $C$. cucurbitina y $C$. dallatorreana se reparten también en Menorca. Por otro lado, no existen datos de captura en la isla de Ibiza, a pesar de que se hayan publicado artículos específicos, tales como el de Hohmann (1984). Esto se debe probablemente a la falta de trabajos de campo. La presencia de $C$. cyanea y $C$. dentiventris en Mallorca, mencionada por Ceballos (1956) y reiterada por Espeso \& Gayubo (1988), no se ha podido confirmar. $C$. dentiventris puede ser confundida fácilmente con $C$. dallatorreana, presente en Mallorca, pero C. cyanea no se puede confundir con ninguna de las especies que aparecen en esa isla.

En las Islas Canarias, sólo ha sido citada $C$. parvula: 2 hembras de Gran Canaria (1 hembra, Artedara, 14-VIII-86, F. La Roche leg.; 1 hembra, Bco. de Los Vicentes, 14-VIII-86, F. La Roche leg.). Los datos de ambas proceden de Hohmann et al. (1993).

La distribución de $C$. parvula es interesante. Esta especie parece estar restringida al clima termo-mediterráneo, especialmente en Francia, donde está limitada estrictamente a la costa mediterránea (Le Goff \& Terzo, 1999). Su presencia en la provincia de Madrid, mencionada por Dusmet (1923), nunca se había confirmado hasta ahora (1 hembra, El Pardo, Sierra de Guadarrama, UTM: 30TVK3686, 24/30-VI-1991, Nieves \& Rey leg., Museo Nacional de Ciencias Naturales de Madrid) y puede sugerir una distribución mucho más amplia que la que hasta ahora se ha intuido.

La fauna ibérica de Ceratina está constituida principalmente por las especies de tres rangos biogeográficos diferentes. El primer grupo se caracteriza por especies de distribución circunmediterránea; incluye solamente a $C$. cucurbitina, C. parvula y C. chalybea. El segundo grupo se caracteriza por especies que aparecen sólo o principalmente en países de la cuenca norte del Mediterráneo; incluye a las siguientes: $C$. chalcites, C. cyanea, $C$. dallatorreana, $C$. dentiventris y $C$. nigrolabiata. En ese grupo, algunas especies se adentran más o menos en el norte de África (C. cyanea, $C$. dallatorreana, $C$. dentiventris), pero son bastante raras. El tercer grupo lo componen especies que se distribuyen fundamentalmente en el norte de África pero que se adentran más o menos en Europa occidental. Incluye a C. albosticta, que alcanza España sólo en una localidad (Cáceres: Mérida, UTM: 29SQD2219, 29/30-VI-1968, J. M. F. Verhoeff leg., Nationaal Natuurhistorisch Museum, Leiden), C. saundersi, que alcanza España y el sur de Italia, y C. callosa y C. mocsar$y i$, que llegan hasta el sur de Francia y el norte de
Italia (Terzo \& Rasmont, 1998). La presencia de $C$. albosticta en España debe ser confirmada. Por otra parte, la distribución de $C$. gravidula es particular (Terzo \& Rasmont, 1996): está restringida a los Balcanes, Italia, Francia, y tiene su localidad conocida más occidental en la costa atlántica portuguesa (ver más arriba).

De la gráfica de fenología (fig. 47) se desprende claramente que las especies del género Ceratina presentan una clara estacionalidad centrada en el verano. Comparadas las curvas con las obtenidas a partir de la fauna francesa (Terzo \& Rasmont, 1993), se aprecian algunas diferencias:

- la primera es que el ciclo en la fauna íbero-balear es más largo: los primeros individuos aparecen en Francia en abril, mientras que en nuestra fauna ya se encuentran activos en marzo; asimismo, las últimas hembras se encuentran activas en Francia en octubre, mientras que en el área íbero-balear aún es posible ver algunas en noviembre.

- la segunda es el máximo de actividad de los machos: en Francia tiene lugar en el mes de julio (coincidiendo con el de las hembras), mientras que en nuestra fauna se adelanta a junio, un mes antes del de las hembras.

En este sentido, la población ibérica de Ceratina se ajusta a la estación principal de floración, que en la Península también se da un mes antes que en Francia. Las causas fundamentales son climatológicas, al tratarse de un país más meridional, con temperaturas medias más altas y mayor número de horas de sol.

\section{AGRADECIMIENTOS}

Los autores agradecen sinceramente a los conservadores de las instituciones y museos y a los propietarios de las colecciones privadas mencionados en el apartado de Material y Métodos por su amabilidad al facilitarnos el estudio de sus colecciones entomológicas, bien en visitas personales, bien gracias al préstamo de material. Igualmente, se agradece a los dos evaluadores anónimos las importantes aportaciones realizadas al manuscrito.

Parte de este trabajo ha sido financiado por el FNRS, por la Fondation Agathon De Potter (Académie Royale des Sciences, des Lettres et des Beaux-Arts de Belgique) y por una beca Germaine Cousin (Société entomologique de France).

\section{Referencias}

Barbier, Y. \& Rasmont, P., 2000. Carto Fauna-Flora, cartographie des données biologiques, logiciel MSDOS version 2.0. Université de Mons-Hainaut. Mons. 
Ceballos, G., 1956. Catálogo de los Himenópteros de España. Trabajos del Instituto Español de Entomología (C.S.I.C.). Madrid. 554 pp.

DALY, H. V., 1983. Taxonomy and ecology of Ceratinini of North Africa and the Iberian Peninsula (Hymenoptera: Apoidea). Systematic Entomology, 8: 29-62.

DinIZ, M. A., 1960. Estado actual do conhecimento dos himenópteros portugueses. Las Ciencias, 25: 211-224.

Dusmet, J. M., 1923. Los Ápidos de España. VI, Géneros Xylocopa Latr. y Ceratina Latr. Memorias de la Real Sociedad Española de Historia Natural, 11: 269-304.

Dusmet, J. M., 1931. Contribución al estudio de los Himenópteros de Portugal. Memórias e Estudos do Museu Zoológico da Universidade de Coimbra, Serie 1, 52: 1-9.

Espeso, M. A. \& Gayubo, S. F., 1988. Apidofauna de la Provincia de Segovia (Hymenoptera: Apoidea). Acta Salamanticensia, 66: 1-81.

Friese, H., 1896. Monographie der Bienengattung Ceratina (Latr.) (Paläarktische Formen). Természetrajzt Füzetek, 19: 34-65.

Gayubo, S. F., Torres, F. \& Heras, C., 1989. Estudio sobre las abejas de la Sierra de Gredos (Hymenoptera, Apoidea). Cuadernos Abulenses, 11: 83-166.

Heras, C. \& Gayubo, S. F., 1989. Contribución al conocimiento de los apoideos de la provincia de Zamora. I. Anthophoridae (Hymenoptera: Apoidea). Eos, 65(1): 61-71.

HohmanN, H., 1984. 15. Bees and wasps on Eivissa: faunistic notes on Hymenoptera Aculeata. In: H. Kuhbier, J.A. Alcover, \& Guerau d'Arellano Tur (eds.). Biogeography and Ecology of the Pityusic Islands. Dr W. Junk Publishers. The Hague, Boston, Lancaster: 303-341.

Hohmann, H., La Roche, F., Ortega, G. \& Barquín, J., 1993. Bienen, Wespen und Ameisen der Kanarischen Inseln (Insecta: Hymenoptera: Aculeata). Veröffentlichungen Übersee-Museum Bremen (Naturwissenschaftlichen), 12(1-2): 14-712.

Le Goff, G. \& Terzo, M., 1999. Nouvelles observations sur Ceratina parvula Smith en France et péninsule Ibérique (Hymenoptera, Xylocopinae). Bulletin de la Société entomologique de France, 104(1): 53-58.

Michener, C. D., 2000. The Bees of the World. The Johns Hopkins University Press. Baltimore and London. viii + 913 pp.

Ornosa, C., 1993. Sobre los Apoidea ibéricos de la Región de Extremadura. Fams. Anthophoridae y Apidae (Hymenoptera). Boletín de la Asociación española de Entomología, 17(2): 165-178.

Ornosa, C. \& Martínez, M. D., 1996. Apoidea de la Cuenca Occidental Alta del Duero (España). Familias Melittidae, Megachilidae, Anthophoridae y Apidae (Hymenoptera). Boletín de la Asociación española de Entomología, 20(3-4): 93-106.
PÉREZ-Í̃̃IGO, C., 1980. Notas sobre los ápidos (Hym., Apoidea) de los Montes Universales, Sierra de Cuenca y Albarracín (provincias de Cuenca y Teruel). Boletín de la Asociación española de Entomología, 4: 43-49.

PÉrez-ÍÑ̃go, C., 1982. Los Ápidos de la Sierra de Guadarrama. I. Fams. Melittidae y Anthophoridae (Hymenoptera, Apoidea). Graellsia, 38: 43-65.

PÉrez-ÍÑIGO, C. \& GAyubo, S. F., 1989. Notas sobre los apoideos de la sierra de Béjar. I. Anthophoridae (Hym. Apoidea). Studia Oecologica, 6: 313-331.

SAUNDERS, E., 1901. Balearic Insects. Hymenoptera aculeata collected in Majorca and Minorca (March and April, 1900) by E. B. Poulton, Oldfield Thomas, and R. I. Pocock, with descriptions of new species. The Entomologist Monthly Magazine, 12(37): 208-211.

Terzo, M., 1998. Annotated list of the species of the genus Ceratina (Latreille) occuring in the Near East, with descriptions of new species (Hymenoptera: Apoidea: Xylocopinae). Linzer biologische Beiträge, 30(2): 719-743.

Terzo, M. \& Rasmont, P., 1993. Les cératines de France (Hymenoptera, Apoidea, Xylocopinae: Ceratina Latreille). In: J. Lhonoré, H. Maurin, R. Guilbot \& P. Keith (eds.). Inventaire et cartographie des invertébrés comme contribution à la gestion des milieux naturels français. Secrétariat de la Faune et de la Flore, Collection patrimoines Naturels, vol. 13: 172-176.

Terzo, M. \& Rasmont, P., 1996. Redescription de Ceratina gravidula Gerstaecker, 1869 et de Ceratina nigroaenea Gerstaecker, 1869 (Hymenoptera, Apoidea, Xylocopinae). Bulletin de la Société entomologique de France 101(1): 5-12.

Terzo, M. \& Rasmont, P., 1998. Ceratina zwakhalsi et $C$. verhoeffi, deux nouvelles espèces de la région ouest-paléarctique (Hymenoptera, Apoidea, Xylocopinae). Tijdschrift voor Entomologie, 140 [1997]: 221-236.

Torres, F., Gayubo, S. F. \& Asensio, E., 1989. Efecto de la presión urbana sobre abejas y avispas (Hymenoptera, Aculeata) en Salamanca. V.: Superfamilia Apoidea. Comunicaciones I.N.I.A., Serie Recursos Naturales, 52: 1-49. 